\title{
Determining the Types of Diseases and Emergency Issues in Pilgrims During Hajj: A Literature Review
}

\author{
Shah Murtaza Rashid Al Masud \\ College of Graduate Studies, \\ Universiti Tenaga Nasional, \\ Malaysia
}

\author{
Asmidar Abu Bakar \\ Department of Systems and \\ Networking, \\ Universiti Tenaga Nasional, \\ Malaysia
}

\author{
Salman Yussof \\ College of Computer Science and \\ Information Technology, \\ Universiti Tenaga Nasional, \\ Malaysia
}

\begin{abstract}
Introduction: Every year 2-3 million pilgrims with different background and most of them are elderly from 184 countries in the world congregate in the holy place 'Haram' at Makkah in Saudi Arabia to perform Hajj. During the pilgrimage, they come across a great deal of rough and tough environment, physical hassle and mental stress. Due to the hardship of travel, fluctuation of weather, continuous walking during religious rites at specific time and sites, many pilgrims injury, feel tired, sick, and exhausted. These may also create complications and overburden the physiological functions including heart, chest, abdominal, and kidney of those who suffer from chronic diseases. Besides the problem of diseases, crowds could cause some other significant problems including missing and lost pilgrims, injuries, and even death. Objective: To determine the common health problems e.g. diseases and emergency incidents encountered by pilgrims during Hajj was the main objective of this study. Methods: An extensive literature review to determine the common health problems and emergency incidents during Hajj was conducted through a systematic literature review. Numerous scholarly databases were used to search for articles published related to health problems and emergency incidents during Hajj from 2008 to 2016. Eligible articles included case reports, experimental and nonexperimental studies. Only thirty articles out of two hundred and sixty articles had met the specific inclusion criteria. Results: The analysis revealed that respiratory diseases include pneumonia, influenza, and asthma $(\mathbf{7 3 . 3 3 \%})$ were the main health problems encountered by the pilgrims during Hajj followed by heat stroke or heat attack, sunlight effects $(\mathbf{1 6 . 6 7 \%})$, cardiovascular disease, heart disease $(10 \%)$. The analysis also revealed that emergency incidents include traffic accidents, and trauma was $3.33 \%$. Notwithstanding the information given above, according to the analysis, the common health problems during Hajj are mainly divided into two categories: non-communicable diseases $(62.5 \%)$ and communicable diseases $(37.5 \%)$. IBM's statistical package for the social sciences (SPSS) version 22 was used to analysis the result. Conclusion: Both communicable and non-communicable health issues are the most common health problems encountered by pilgrims during Hajj. But, due to lack of existing studies associated with this research area, a definite conclusion could not be made. However, our findings demonstrated the necessity of new research to find solutions to pilgrims' health problems during Hajj.
\end{abstract}

Keywords-Hajj; pilgrims; health; communicable diseases; non-communicable diseases; emergency

\section{INTRODUCTION}

The largest yearly religious mass gathering worldwide is the Hajj, one of the obligatory five pillars of Islam. Every year during Hajj event an amount of 2-3 million pilgrims and a total of 10 million pilgrims for Hajj and Umrah from 184 countries congregate in Makkah, Saudi Arabia [1, 2, 3, 4]. The number of Hajj pilgrims has increased rapidly from 58, 584 in 1920 to 20, 00,000 (approx) in 2015 where 13, 84, 931 attended from outside Saudi Arabia. During the last 95 years, the increase rate of foreign pilgrims is 3.5 , and the reason is a nonstop expansion of the Grand Mosque at Ka'aba in Makkah [5]. The following Fig. 1 shows the number of pilgrims attending the Hajj during the year 2006-2015. Data were retrieved from the official portal of the royal embassy of Saudi Arabia, Saudi Ministry of Hajj, and central department of statistics and information $[5,6,7]$.

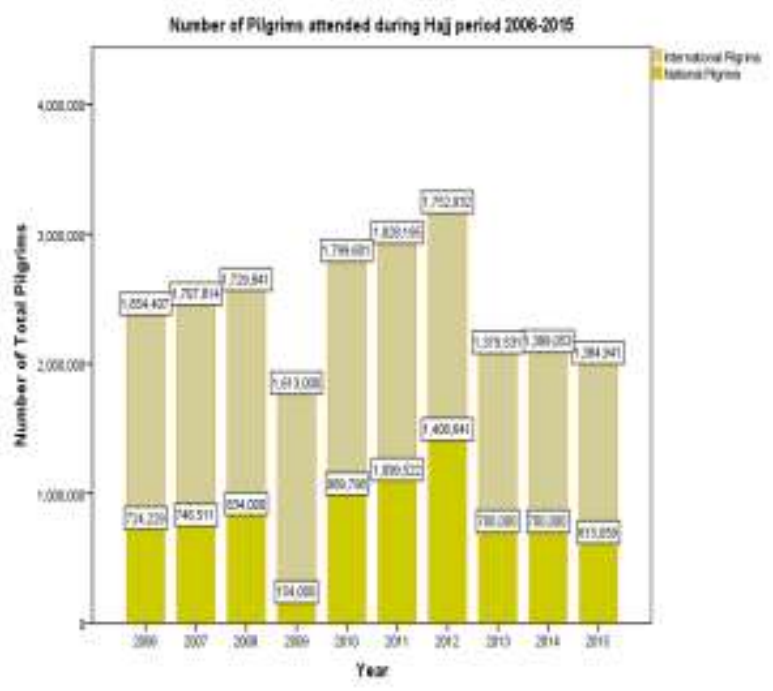

Fig. 1. Statistics of number of pilgrims performed Hajj during the year 2006-2016

Hajj is a dynamic system to appreciate fully the physical dimensions of it which imply movement and travel. Walking Hajj distance: from Kaaba to Mina: $8 \mathrm{~km}$, from Mina to Arafat: $13 \mathrm{~km}$, Arafat to Muzdalifah: $13 \mathrm{~km}$, Muzdalifah to 
Mina: $2 \mathrm{~km}$, Mina to Jamarat Akabah: $3.8 \mathrm{~km}$. Makkah to Madina $450 \mathrm{~km}$. On arrival, the pilgrims must don the Ihram and circumambulate the Ka'ba seven times and then perform the Saa'y seven times between the hills Safa and Marwah, a total distance $3.5 \mathrm{~km}$. Crowd densities can increase to seven individuals per $\mathrm{m}^{2}$ during Hajj. Overcrowding is one of the major problems since the area is limited and the numbers are increasing annually. The area of pilgrimage rites is a sandy valley embraced by rugged sun-baked mountains. In Makkah, especially during the hot months of May to September the temperature ranges between $38^{\circ} \mathrm{C}$ and $50^{\circ} \mathrm{C}$ with a relative humidity of $25 \%$ to $50 \%$ [8]. This kind of hot environment with high radiant heat favors the development of heat illnesses e.g. heat exhaustion, heat stroke, unintentional physical injuries, and respiratory illnesses, dehydration called as noncommunicable diseases or problems [9].

The imminence between pilgrims due to the packed and crowded accommodation, congregation, and prayers creates an ideal atmosphere for spread and transmission of infectious diseases. Influenza, influenza-like illness, meningococcal disease, viral hemorrhagic fevers, yellow fever, cholera, polio, plague, tuberculosis and gastrointestinal infections, foodborne diseases e.g. diarrhea, food poisoning, etc. are examples of communicable diseases $[10,11,12,13]$. Due to the physical exertion and overcrowding situation, some pre-existing chronic diseases such as asthma, heart disease, chronic chest conditions, diabetes, renal and liver disease may become harmful for pilgrims especially elderly which eventually favors the spread of communicable namely non-chronic diseases or infectious disease [14].

According to the findings, respiratory diseases includes pneumonia, influenza, and asthma $73.33 \%$ were the main health problems encountered by the pilgrims during Hajj followed by heat stroke/ attack, sunlight effects $16.67 \%$, diabetic/ diabetes mellitus $13.33 \%$. Cardiovascular disease, heart disease $10 \%$, hypertension $6.67 \%$, dehydration $6.67 \%$, musculoskeletal $6.67 \%$, urinary tract problems $3.33 \%$, meningococcal disease $3.33 \%$, diarrhea and jaundice $3.33 \%$, finally, traffic accidents and trauma $3.33 \%$. According to the studies, the pilgrims encountered $62.5 \%$ non-communicable diseases and problems along with $37.5 \%$ communicable diseases during Hajj. To determine the common health problems e.g. diseases and emergency incidents encountered by pilgrims during Hajj was the primary objective of this study. Hence, the result from this study could be beneficial in initiating, planning and design the appropriate healthcare system to prevent diseases and emergency situations encountered by Hajj pilgrims.

\section{SEARCH StRATEGY AND SELECTION CRITERIA}

The Lancet, IEEE Explore, MedLine, EBSCO Host, PubMed, Google Scholar, Science Direct, the Elsevier (Scopus), Academic search complete, Springer Link, ACM digital library, Emerald Insight, Taylors and Francis and Wiley database were used to search for articles published related to health problems and emergency incidents during Hajj from 2008 to 2016. The majority of related articles were published in various prominent journals around the world where most of the studies were carried in Saudi Arabia, Iran,
France, United Kingdom, Malaysia, and Pakistan. Finally, we accessed official Saudi governmental statistics, with a particular emphasis on data from the Saudi Ministry of Health and Saudi Ministry of Hajj and Umrah. The combinations of specific keywords were utilized to retrieve the articles including pilgrims, Hajj, Diseases, healthcare, health problem, medical, medicine, overcrowd, emergency incidents, health pattern. Only thirty studies had met the specific inclusion criteria: the subjects were Hajj, pilgrims, health, crowd, emergency; the type of study was experimental or nonexperimental study; available full article in English. The research was conducted to find the answers to these questions e.g. What are the major diseases do the pilgrims carry and suffering? What are the reasons behind emergency incidents during Hajj? What are the emergency incidents occur during Hajj? Fig. 2 illustrates the search strategy as depicted. This paper is organized as, Section II: Search strategy and selection criteria; Section III: Data collection and analysis; Section IV: Analyzing the result; Section V: Discussion of the research; and Section VI: Conclusion.

\section{DATA COLLECTION AND ANALYSIS}

Items and their distributions of all respondents in the studies chosen were well briefed and summarized according to the author, and year. Meanwhile, the subsequent information gathered were objective, study design, the pattern of health problems and emergency situations, analyzing of the result and discussion of research shown in Table I. Table I tabulated all the information from selected articles.

\section{ANALYSING THE RESUlt}

Universally, elderly pilgrims were vulnerable to get the infection due to decreased rate of immune responses which is actively provoked by other factors such as hard work, lack of sleep and disturbances in the dietary schedule, and mental stress. Main reasons for infectious disease transmission due to airborne agents and pilgrims health hazards; especially injuries, trauma, etc. are extended stays at Hajj sites, and physical exhaustion, extreme heat, and crowded accommodation [15]. The most feared trauma hazard during Hajj is stampede causing huge casualties. Chronicle of Hajj disasters as depicted in Table II, where data retrieved from the official portal of the embassy of Saudi Arabia, Saudi Ministry of Hajj, and central department of statistics and information [5, 6, and 7]. Hajjis face multiple health issues like extreme temperatures, intravascular volume and electrolytes disturbances which also increases the risk of communicable and non-communicable diseases, where Hajj pilgrims encounter a great deal of tough physical and mental stress [16].

Some studies related to the current health and diseases situation during Hajj spotted that, one in three pilgrims experiences such respiratory symptoms [17]. Congestion and close contact stimulate the spread of infection especially upper respiratory tract infections URTIs followed by diseases of the skin, GIT, rheumatology [18, 19], and URTIs as reported among the most common cause of illness among Iranian pilgrims [20]. Other hazards include traffic accidents and fire injuries are reported in the literature [21]. Due to the hazards like accidents, overcrowding situation, and human jam many 
pilgrims divert from their groups and get lost, where pilgrims are walking shoulder to shoulder in such massive Hajj gathering.

Research Objective

To determine the common health problems (diseases) and emergency incidents encountered by pilgrims during Haii

Research Questions

What are the major diseases do the pilgrims carry and suffering from?

What are the reasons behind emergency incidents during Hajj?

What are the emergency incidents occur during Hajj?

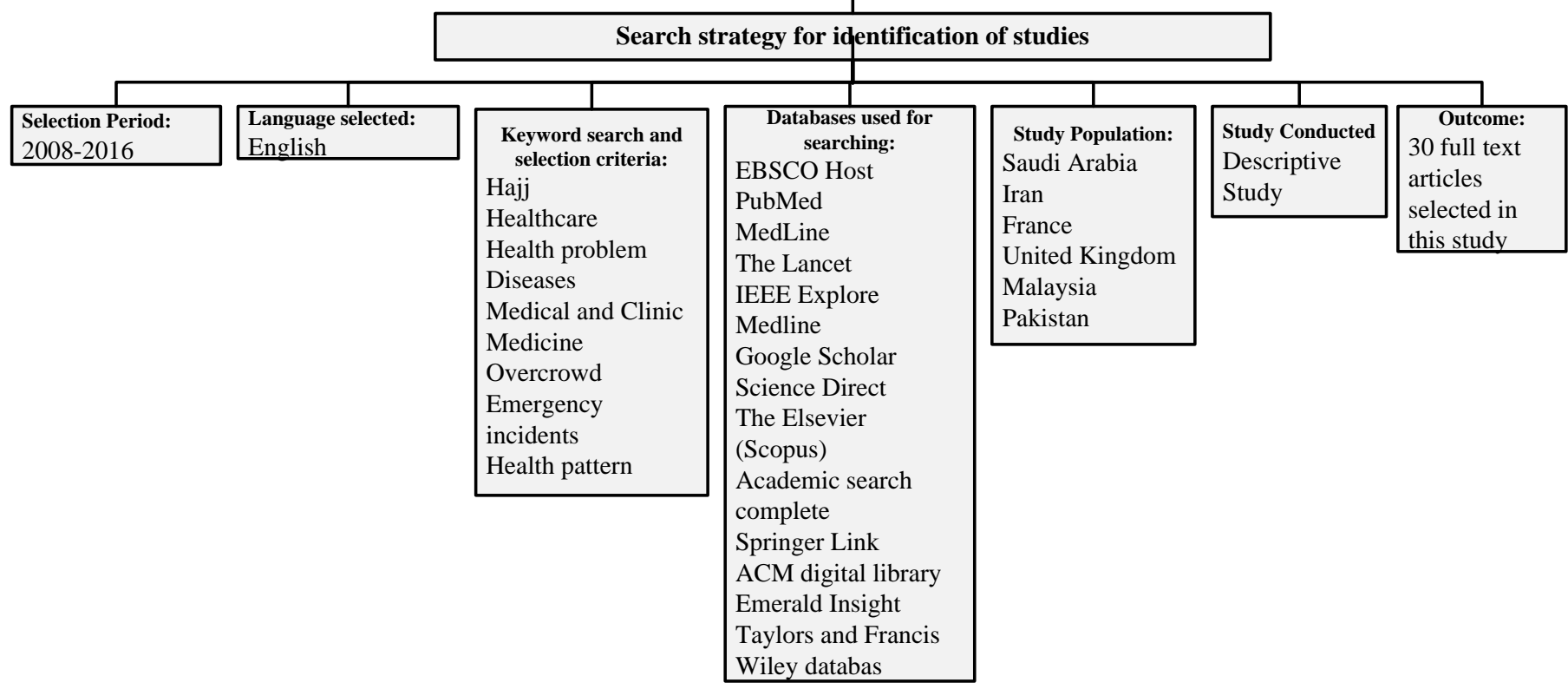

Fig. 2. Flow of the search strategy

TABLE I. EXPERIMENTAL StUdy ON THE BASEd ON PILGRIMS’ HeAlth PROBLEMS AND EMERGENCY INCIDENTS DURING HAJJ

\begin{tabular}{|c|c|c|c|}
\hline $\begin{array}{l}\text { Research } \\
\text { Author, } \\
\text { Year } \\
\text { [Ref] }\end{array}$ & Objectives & Method & $\begin{array}{l}\text { Result and } \\
\text { Discussion }\end{array}$ \\
\hline $\begin{array}{l}\text { Memish et } \\
\text { al. (2016) } \\
\text { [22] }\end{array}$ & $\begin{array}{l}\text { Hajj health } \\
\text { services } \\
\text { emerged as an ad- } \\
\text { hoc activity to } \\
\text { treat } \\
\text { ailing pilgrims } \\
\text { which are subject } \\
\text { to consultation } \\
\text { with global } \\
\text { stakeholders for } \\
\text { compliance with } \\
\text { the International } \\
\text { Health } \\
\text { Regulations. }\end{array}$ & $\begin{array}{l}\text { Comparative } \\
\text { study }\end{array}$ & $\begin{array}{l}\text { Emphasized on } \\
\text { non-communicable } \\
\text { diseases (NCDs) } \\
\text { prevention }\end{array}$ \\
\hline $\begin{array}{l}\text { Bader et } \\
\text { al. }(2016) \\
{[23]}\end{array}$ & $\begin{array}{l}\text { Clinical pattern of } \\
\text { pneumonia during } \\
\text { the Hajj period } \\
2004-2013\end{array}$ & $\begin{array}{l}\text { A } \\
\text { retrospective } \\
\text { cohort } \\
\text { analysis }\end{array}$ & $\begin{array}{l}\text { The mortality rate } \\
\text { in the intensive care } \\
\text { unit-ICU was } \\
21.45 \% \text {, while the } \\
\text { rate in the ward was } \\
2.4 \% \text {. }\end{array}$ \\
\hline $\begin{array}{l}\text { Murtaza } \\
\text { et al. } \\
\text { (2015) }\end{array}$ & $\begin{array}{l}\text { Pattern of diseases } \\
\text { among pilgrims } \\
\text { seeking medical }\end{array}$ & $\begin{array}{l}\text { Observationa } \\
1 \text { study }\end{array}$ & $\begin{array}{l}\text { Respiratory diseases } \\
52.5 \% \text {, } \\
\text { musculoskeletal }\end{array}$ \\
\hline
\end{tabular}

\begin{tabular}{|c|c|c|c|}
\hline [24] & services & & $\begin{array}{l}29.6 \%, \text { skin } 5.9 \%, \\
\text { cardiovascular } \\
2.9 \%, \text { urinary tract } \\
\text { problems } 2.3 \% \text {, } \\
\text { diabetic } 17.9 \% \text { and } \\
26.4 \% \text { hypertensive. }\end{array}$ \\
\hline $\begin{array}{l}\text { Sindy et } \\
\text { al. }(2015) \\
{[25]}\end{array}$ & $\begin{array}{l}\text { To assess the } \\
\text { pattern of patients } \\
\text { and illnesses } \\
\text { encountered at one } \\
\text { health facility at } \\
\text { Arafat during Hajj } \\
2013 \text { where acute } \\
\text { severe asthma and } \\
\text { injuries were the } \\
\text { major problems } \\
\text { encountered }\end{array}$ & $\begin{array}{l}\text { Cross- } \\
\text { sectional } \\
\text { study }\end{array}$ & $\begin{array}{l}\text { Bronchial Asthma } \\
34 \% \text {, } \\
\text { Injuries } 16 \% \text {, } \\
\text { Diabetes Mellitus } \\
\text { 10.4\%, Heat effects } \\
6.6 \% \text {, Hypertension } \\
6.6 \% \text {, } \\
\text { Gastroenteritis } \\
6.63 \% \\
\text { Dehydration } 5.69 \%\end{array}$ \\
\hline $\begin{array}{l}\text { Habsah et } \\
\text { al. (2015) } \\
\text { [26] }\end{array}$ & $\begin{array}{l}\text { Determining the } \\
\text { consequence of } \\
\text { influenza } \\
\text { vaccination } \\
\text { alongside acute } \\
\text { respiratory illness } \\
\text { amongst } \\
\text { Malaysian Hajj } \\
\text { pilgrims }\end{array}$ & $\begin{array}{l}\text { Observationa } \\
1 \text { cohort } \\
\text { study }\end{array}$ & $\begin{array}{l}\text { Respiratory illness } \\
\text { was a major } \\
\text { problem and caused } \\
\text { high hospital } \\
\text { admission during } \\
\text { Hajj seasons. }\end{array}$ \\
\hline $\begin{array}{l}\text { Abdulrah } \\
\text { man et al. } \\
\text { (2015) }\end{array}$ & $\begin{array}{l}\text { Evaluating the } \\
\text { diseases pattern } \\
\text { among pilgrims } \\
\text { during the Hajj }\end{array}$ & $\begin{array}{l}\text { Cross } \\
\text { sectional } \\
\text { study }\end{array}$ & $\begin{array}{l}\text { Respiratory } \\
\text { problems } 17.6 \% \text {, } \\
\text { skin diseases } \\
15.7 \% \text {, }\end{array}$ \\
\hline
\end{tabular}




\begin{tabular}{|c|c|c|c|}
\hline [27] & season 2013 & & $\begin{array}{l}\text { gastrointestinal tract } \\
\text { (GIT) diseases } \\
13.2 \%\end{array}$ \\
\hline $\begin{array}{l}\text { Nurul et } \\
\text { al. }(2014) \\
{[28]}\end{array}$ & $\begin{array}{l}\text { Overcrowding, } \\
\text { extreme } \\
\text { temperatures, and } \\
\text { electrolytes } \\
\text { imbalance are } \\
\text { common among } \\
\text { pilgrims. These } \\
\text { factors trigger the } \\
\text { increased risk for } \\
\text { the communicable } \\
\text { and non- } \\
\text { communicable } \\
\text { disease. }\end{array}$ & $\begin{array}{l}\text { Cross- } \\
\text { sectional } \\
\text { study }\end{array}$ & $\begin{array}{l}\text { The analysis } \\
\text { revealed that } \\
\text { respiratory diseases } \\
76.2 \% \text { was the main } \\
\text { health problem } \\
\text { followed by skin } \\
\text { disease } 7.4 \% \text {, } \\
\text { meningococcal } \\
\text { disease } 3.7 \% \text { and } \\
\text { heat stroke } 3.7 \% \text {. }\end{array}$ \\
\hline $\begin{array}{l}\text { Memish et } \\
\text { al. }(2014) \\
\text { [29] }\end{array}$ & $\begin{array}{l}\text { Infectious disease } \\
\text { surveillance } \\
\text { during Hajj } 2012 \\
\text { and } 2013\end{array}$ & $\begin{array}{l}\text { Descriptive } \\
\text { study }\end{array}$ & $\begin{array}{l}\text { Report showed that } \\
\text { infectious diseases } \\
\text { posed the major } \\
\text { thread among } \\
\text { pilgrims }\end{array}$ \\
\hline $\begin{array}{l}\text { Habida et } \\
\text { al. (2014) } \\
{[30]}\end{array}$ & $\begin{array}{l}\text { Need for equal } \\
\text { attention on } \\
\text { infectious and } \\
\text { non- } \\
\text { communicable } \\
\text { diseases during } \\
\text { Hajj }\end{array}$ & $\begin{array}{l}\text { Comparative } \\
\text { study }\end{array}$ & $\begin{array}{l}\text { The majority about } \\
60 \% \text { of pilgrims } \\
\text { participating in the } \\
\text { Hajj are elderly } \\
\text { with preexisting } \\
\text { chronic conditions. } \\
\text { Mental stress, } \\
\text { overcrowded } \\
\text { conditions, extreme } \\
\text { heat and sunlight } \\
\text { leading to heat } \\
\text { cramps, heat } \\
\text { exhaustion, heat } \\
\text { stroke, and } \\
\text { dehydration which } \\
\text { ultimately favors } \\
\text { spreading infectious } \\
\text { diseases. }\end{array}$ \\
\hline $\begin{array}{l}\text { Osamah et } \\
\text { al. (2014) } \\
\text { [31] }\end{array}$ & $\begin{array}{l}\text { The attack rate of } \\
\text { influenza-like } \\
\text { illnesses due to } \\
\text { respiratory viruses } \\
\text { among the } \\
\text { pilgrims from } \\
\text { Saudi Arabia, } \\
\text { Australia, and } \\
\text { Qatar during Hajj } \\
2013\end{array}$ & $\begin{array}{l}\text { Cross- } \\
\text { sectional } \\
\text { study }\end{array}$ & $\begin{array}{l}11 \% \text { pilgrims had } \\
\text { influenza, where } \\
\text { rhinovirus was the } \\
\text { commonest cause } \\
28 \% \text {. }\end{array}$ \\
\hline $\begin{array}{l}\text { Samir et } \\
\text { al. (2014) } \\
{[32]}\end{array}$ & $\begin{array}{l}\text { Specimen of } \\
\text { France pilgrims } \\
\text { during Hajj } 2013 \\
\text { were tested for } \\
\text { respiratory viruses } \\
\text { and bacteria }\end{array}$ & $\begin{array}{l}\text { Prospective } \\
\text { cohort study }\end{array}$ & $\begin{array}{l}\text { Rhinovirus (14.0\%), } \\
\text { coronavirus E229 } \\
(12.4 \%) \text {, and } \\
\text { influenza A (H3N2) } \\
\text { virus }(6.2 \%)\end{array}$ \\
\hline $\begin{array}{l}\text { Samir et } \\
\text { al. }(2013) \\
{[33]}\end{array}$ & $\begin{array}{l}\text { Identifying the } \\
\text { pattern of at least } \\
11 \text { respiratory } \\
\text { viruses during } \\
\text { Hajj } 2012 \text { among } \\
\text { the pilgrims of } \\
\text { France }\end{array}$ & $\begin{array}{l}\text { Prospective } \\
\text { cohort study }\end{array}$ & $\begin{array}{l}38.6 \% \text { pilgrims } \\
\text { were affected by } \\
\text { different respiratory } \\
\text { viruses including } \\
\text { rhinovirus, } \\
\text { adenovirus, } \\
\text { influenza B, and } \\
\text { enterovirus }\end{array}$ \\
\hline $\begin{array}{l}\text { Tawfiq et } \\
\text { al. (2013) } \\
\text { [34] }\end{array}$ & $\begin{array}{l}\text { Causes of } \\
\text { respiratory tract } \\
\text { infection during } \\
\text { Hajj }\end{array}$ & $\begin{array}{l}\text { Cross- } \\
\text { sectional } \\
\text { study }\end{array}$ & $\begin{array}{l}\text { The most common } \\
\text { respiratory tract } \\
\text { viruses are } \\
\text { influenza and } \\
\text { rhinovirus. } \\
\text { Pneumonia is a } \\
\text { significant cause of } \\
\text { an admission }\end{array}$ \\
\hline
\end{tabular}

\begin{tabular}{|c|c|c|c|}
\hline & & & $\begin{array}{l}\text { accounting for } 25- \\
50 \% \text { of such } \\
\text { admission. }\end{array}$ \\
\hline $\begin{array}{l}\text { Abdullah } \\
\text { et al. } \\
(2012) \\
{[35]}\end{array}$ & $\begin{array}{l}\text { Establishing a } \\
\text { pragmatic system } \\
\text { to manage the } \\
\text { challenge of } \\
\text { cardiovascular } \\
\text { morbidities and } \\
\text { mortality during } \\
\text { Hajj. }\end{array}$ & $\begin{array}{l}\text { Cross- } \\
\text { sectional } \\
\text { study }\end{array}$ & $\begin{array}{l}\text { Most significant } \\
\text { causes of death } \\
\text { during } 2002 \text { Hajj } \\
\text { was cardiovascular } \\
\text { diseases (with } \\
\text { hypertension) } \\
45.8 \% \text {, followed by } \\
\text { respiratory and } \\
\text { cardiac system } \\
\text { failure } 14.3 \% \text {, } \\
\text { traffic accidents and } \\
\text { other traumas } 6.4 \%\end{array}$ \\
\hline $\begin{array}{l}\text { Alzahrani } \\
\text { et al. } \\
(2012) \\
{[36]}\end{array}$ & $\begin{array}{l}\text { Seeking medical } \\
\text { services for } \\
\text { pilgrims suffering } \\
\text { from various } \\
\text { diseases. }\end{array}$ & $\begin{array}{l}\text { Descriptive } \\
\text { study }\end{array}$ & $\begin{array}{l}\text { Respiratory diseases } \\
60.8 \% \text {, } \\
\text { musculoskeletal } \\
17.6 \% \text {, skin disease } \\
15.0 \% \text { and } \\
\text { gastrointestinal } \\
13.1 \% \text {. }\end{array}$ \\
\hline $\begin{array}{l}\text { Mandoura } \\
\text { h et al. } \\
(2012) \\
{[37]}\end{array}$ & $\begin{array}{l}\text { Determining } \\
\text { diseases among } \\
\text { hospitals } \\
\text { providing medical } \\
\text { care to Hajj } \\
\text { pilgrims. }\end{array}$ & $\begin{array}{l}\text { Prospective } \\
\text { cohort study. }\end{array}$ & $\begin{array}{l}\text { Pneumonia was the } \\
\text { primary cause of } \\
\text { critical illness } \\
27.2 \%\end{array}$ \\
\hline $\begin{array}{l}\text { Memish et } \\
\text { al. (2012) } \\
\text { [38] }\end{array}$ & $\begin{array}{l}\text { The occurrence of } \\
\text { different } \\
\text { respiratory viruses } \\
\text { among healthcare } \\
\text { worker during } \\
\text { Hajj event. } \\
\text { Pilgrims' } \\
\text { attendance at the } \\
\text { Hajj was } \\
\text { proportional to the } \\
\text { increase of } \\
\text { acquiring } \\
\text { influenza. }\end{array}$ & $\begin{array}{l}\text { Cross- } \\
\text { sectional } \\
\text { study }\end{array}$ & $\begin{array}{l}\text { Rhinoviruses and } \\
\text { Coronaviruses } 229 \mathrm{E} \\
\text { were detected } \\
\text { during the study. } \\
\text { Influenza A 0.1- } \\
0.2 \%\end{array}$ \\
\hline $\begin{array}{l}\text { Moattari } \\
\text { et al. } \\
(2012) \\
{[39]}\end{array}$ & $\begin{array}{l}\text { Seasonal and } \\
\text { pandemic } \\
\text { influenza attack } \\
\text { rate among } \\
\text { returning Iranian } \\
\text { pilgrims after the } \\
2009 \text { Hajj. }\end{array}$ & $\begin{array}{l}\text { Cross- } \\
\text { sectional } \\
\text { study }\end{array}$ & $\begin{array}{l}\text { By virus culture, it } \\
\text { was recorded that } \\
\text { about } 9.1 \% \text { pilgrims } \\
\text { had influenza }\end{array}$ \\
\hline $\begin{array}{l}\text { Almalki et } \\
\text { al. (2012) } \\
\text { [40] }\end{array}$ & $\begin{array}{l}\text { Estimating the } \\
\text { frequency of the } \\
\text { cardiovascular } \\
\text { diseases during } \\
\text { Hajj } 2011 \\
\end{array}$ & $\begin{array}{l}\text { Descriptive } \\
\text { study }\end{array}$ & $\begin{array}{l}\text { Ischemic heart } \\
\text { disease } 34 \%, \\
\text { elevated blood } \\
\text { pressure } 20 \% \text { and } \\
\text { stroke } 17 \% \\
\end{array}$ \\
\hline $\begin{array}{l}\text { Ziyaeyan } \\
\text { et al. } \\
(2012) \\
{[41]} \\
\end{array}$ & $\begin{array}{l}\text { The occurrence of } \\
\text { A (H1N1) among } \\
\text { returning Iranian } \\
\text { pilgrims. }\end{array}$ & $\begin{array}{l}\text { Cross- } \\
\text { sectional } \\
\text { study }\end{array}$ & Influenza A (1.6\%) \\
\hline $\begin{array}{l}\text { Saeed et } \\
\text { al. }(2012) \\
{[42]}\end{array}$ & $\begin{array}{l}\text { The prevalence of } \\
\text { three symptoms of } \\
\text { interest diarrhea, } \\
\text { acute } \\
\text { respiratory } \\
\text { infection and } \\
\text { jaundice among } \\
\text { Afghanistan Hajjis } \\
\text { during Hajj 2010 }\end{array}$ & $\begin{array}{l}\text { Cross- } \\
\text { sectional } \\
\text { Study }\end{array}$ & $\begin{array}{l}\text { Diarrhea and } \\
\text { jaundice remained } \\
\text { constant, but Acute } \\
\text { respiratory infection } \\
1.4 \% \text { at pre-transit } \\
\text { to } 4 \% \text { at transit area } \\
\text { and } 37 \% \text { during } \\
\text { Hajj. }\end{array}$ \\
\hline $\begin{array}{l}\text { Asghar et } \\
\text { al. } \\
(2011) \\
{[43]}\end{array}$ & $\begin{array}{l}\text { Find the common } \\
\text { causes of bacterial } \\
\text { pneumonia during } \\
\text { the } 2005 \text { Hajj } \\
\text { season. }\end{array}$ & $\begin{array}{l}\text { Cross- } \\
\text { sectional } \\
\text { study }\end{array}$ & $\begin{array}{l}\text { Clinically suspected } \\
\text { pneumonia } 53.9 \% \\
\text { were confirmed } \\
\text { positive. }\end{array}$ \\
\hline
\end{tabular}




\begin{tabular}{|c|c|c|c|}
\hline $\begin{array}{l}\text { Mimish et } \\
\text { al. } \\
(2011) \\
{[44]}\end{array}$ & $\begin{array}{l}\text { Electrocardiograp } \\
\text { hic (ECG) changes } \\
\text { in exposed to high } \\
\text { outdoor } \\
\text { temperature 45- } \\
\text { degree centigrade, } \\
\text { humidity } \\
\text { approaching } 80 \% \\
\text { with heat stroke } \\
\text { and heat } \\
\text { exhaustion. }\end{array}$ & $\begin{array}{l}\text { Case-control } \\
\text { study }\end{array}$ & $\begin{array}{l}\text { Sinus tachycardia } \\
\text { and ischemic } \\
\text { changes. }\end{array}$ \\
\hline $\begin{array}{l}\text { Mirza et } \\
\text { al. } \\
(2011) \\
{[45]}\end{array}$ & $\begin{array}{l}\text { Predictors of } \\
\text { asthma } \\
\text { exacerbations } \\
\text { during the Hajj. }\end{array}$ & $\begin{array}{l}\text { Cohort } \\
\text { study. }\end{array}$ & $\begin{array}{l}46.6 \% \text { suffered } \\
\text { from mild asthma } \\
\text { attack, and } 31 \% \\
\text { suffered from } \\
\text { moderate asthma } \\
\text { attack }\end{array}$ \\
\hline $\begin{array}{l}\text { Zakuan et } \\
\text { al. } \\
(2010) \\
{[46]}\end{array}$ & $\begin{array}{l}\text { The relationship } \\
\text { between pre- } \\
\text { morbid conditions } \\
\text { and influenza-like } \\
\text { illness (ILI) } \\
\text { among Malaysian } \\
\text { pilgrims during } \\
\text { Hajj } 2007\end{array}$ & $\begin{array}{l}\text { Cross- } \\
\text { sectional } \\
\text { study }\end{array}$ & $\begin{array}{l}\text { Underlying asthma } \\
\text { was significantly } \\
\text { associated with } \\
\text { severe ILI, e.g. a } \\
\text { sore throat, longer } \\
\text { duration of a cough, } \\
\text { runny nose } \\
\text { ( } \mathrm{p}=0.016 \text { ) and the } \\
\text { Pilgrims who } \\
\text { suffered from } \\
\text { chronic obstructive } \\
\text { pulmonary diseases } \\
\text { (COPD), diabetes } \\
\text { mellitus and } \\
\text { respiratory problem. }\end{array}$ \\
\hline $\begin{array}{l}\text { Alborzi et } \\
\text { al. } \\
(2009) \\
{[47]}\end{array}$ & $\begin{array}{l}\text { Incidence of the } \\
\text { common } \\
\text { respiratory viruses } \\
\text { among Iranian } \\
\text { pilgrims during } \\
\text { Hajj } 2006\end{array}$ & $\begin{array}{l}\text { Cross- } \\
\text { sectional } \\
\text { study }\end{array}$ & $\begin{array}{l}\text { The rates of } \\
\text { different types of } \\
\text { respiratory virus } \\
\text { infections: influenza } \\
(9.8 \%), \\
\text { parainfluenza } \\
(7.4 \%) \text {, adenovirus } \\
(5.4 \%) \text { and RSV } \\
(1.4 \%) \text {. Where } \\
\text { Viral agents }(25 \%) \\
\text { of nasal specimens }\end{array}$ \\
\hline $\begin{array}{l}\text { Gautret et } \\
\text { al. } \\
(2009) \\
{[48]}\end{array}$ & $\begin{array}{l}\text { The incidence of a } \\
\text { febrile cough } \\
\text { among French } \\
\text { pilgrims during } \\
\text { Hajj. }\end{array}$ & $\begin{array}{l}\text { Prospective } \\
\text { cohort study }\end{array}$ & $\begin{array}{l}\text { Diabetes mellitus } \\
22.8 \% \text {, } \\
\text { Hypertension } 25.3\end{array}$ \\
\hline $\begin{array}{l}\text { Gautret et } \\
\text { al. } \\
(2009) \\
{[49]}\end{array}$ & $\begin{array}{l}\text { Investigate } \\
\text { prospectively the } \\
\text { occurrence of } \\
\text { common health } \\
\text { hazard in } \\
\text { French pilgrims } \\
\text { during Hajj } 2007 \\
\end{array}$ & $\begin{array}{l}\text { Prospective } \\
\text { cohort study }\end{array}$ & $\begin{array}{l}\text { Cough } 51 \% \text {, } \\
\text { followed by a } \\
\text { headache, heat } \\
\text { stress, and fever }\end{array}$ \\
\hline $\begin{array}{l}\text { Rashid et } \\
\text { al. } \\
(2008) \\
{[50]}\end{array}$ & $\begin{array}{l}\text { Burden of } \\
\text { influenza and RSV } \\
\text { in asymptomatic } \\
\text { British pilgrims } \\
\text { during Hajj }\end{array}$ & $\begin{array}{l}\text { Cross- } \\
\text { sectional } \\
\text { study }\end{array}$ & $\begin{array}{l}\text { Influenza or RSV } \\
\text { with } 18 \%\end{array}$ \\
\hline $\begin{array}{l}\text { Rashid et } \\
\text { al. } \\
(2008) \\
{[51]}\end{array}$ & $\begin{array}{l}\text { Rates of various } \\
\text { influenza virus } \\
\text { infections among } \\
\text { the UK and Saudi } \\
\text { pilgrims during } \\
\text { Hajj. }\end{array}$ & $\begin{array}{l}\text { Comparative } \\
\text { study }\end{array}$ & $\begin{array}{l}25 \% \text { the UK and } \\
\text { Saudi pilgrims }\end{array}$ \\
\hline
\end{tabular}

A total of 30 studies related to communicable diseases or infectious diseases and non-communicable diseases/ problems or chronic diseases were used for analyzing the result. Regarding the study design of the selected articles; most of the studies were based on cross-sectional studies 46.67\%, prospective cohort study $16.67 \%$, descriptive study $10 \%$, comparative study $6.67 \%$, retrospective cohort analysis $3.33 \%$, comparative study $3.33 \%$, case-control study $3.33 \%$, and Cohort study 3.33\% as depicted in Fig. 3. Different types of instruments were used in the quantitative studies.

\section{TABLE II. CHRONICLE OF DISASTER: 1990-2015}

1990: 1426 pilgrims killed by stampede/asphyxiation in tunnel leading to holy sites

1994: 270 killed in a stampede

1997: 343 pilgrims died and 1500 injured in a fire

1998: 119 pilgrims died in a stampede

2001: 35 pilgrims died in a stampede

2003: 14 pilgrims died in a stampede

2004: 251 pilgrims died in a stampede

2006: 76 pilgrims died after a hotel housing pilgrims collapsed; a stampede wounded 289 , killing 380

2015: 4173 pilgrims died in a stampede

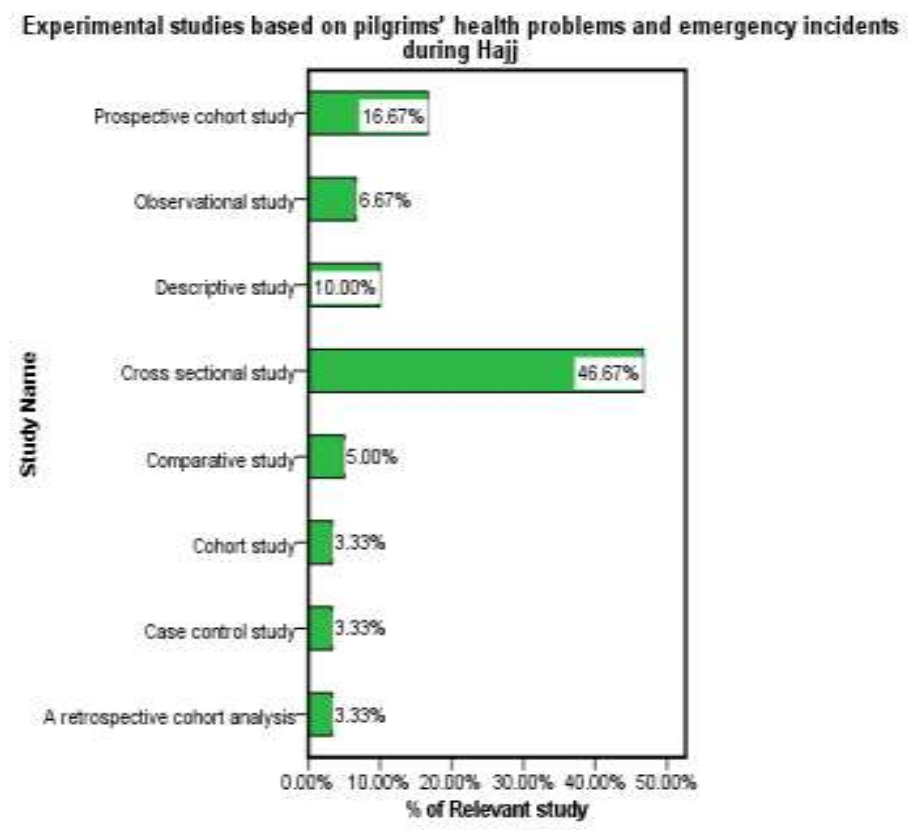

Fig. 3. Percentage of studies based on pilgrims' health problems and emergency incidents during Hajj

Out of 30 selected studies, 22 articles had concluded that respiratory diseases include pneumonia, influenza, and asthma $73.33 \%$ were the main health problems encountered by the pilgrims during Hajj as presented in Table III and Fig. 4. The above findings followed by heat stroke/ attack, sunlight effects $16.67 \%$, diabetic/ diabetes mellitus $13.33 \%$, cardiovascular disease, heart disease $10 \%$, hypertension $6.67 \%$, dehydration $6.67 \%$, musculoskeletal $6.67 \%$, urinary tract problems $3.33 \%$, meningococcal disease $3.33 \%$, diarrhea and jaundice $3.33 \%$, finally, traffic accidents and trauma $3.33 \%$. 
TABLE III. Major Health Problems Encountered By PILgRIMS ACCORDING TO THE STUDIES AND CATEGORIZATION OF DISEASES

\begin{tabular}{|c|c|c|c|}
\hline $\begin{array}{l}\text { Diseases name, } \\
\text { major health } \\
\text { problems }\end{array}$ & $\begin{array}{l}\text { Number of } \\
\text { studies }\end{array}$ & $\begin{array}{l}\text { Percentage } \\
\text { of health } \\
\text { problems }\end{array}$ & $\begin{array}{l}\text { Category based } \\
\text { on } \\
\text { Communicable } \\
\text { Diseases } \\
\text { (CD)/problems- } \\
\text { Chronic, Non- } \\
\text { Communicable } \\
\text { Diseases(NCD)- } \\
\text { Infectious }\end{array}$ \\
\hline $\begin{array}{l}\text { Respiratory } \\
\text { disease includes } \\
\text { asthma, influenza, } \\
\text { pneumonia }\end{array}$ & $\begin{array}{l}{[23][24][26]} \\
{[27][28][31]} \\
{[32][33][34]} \\
{[35][36][37]} \\
{[38]} \\
{[39][41][42]} \\
{[43][45][46]} \\
{[47][50][51]}\end{array}$ & $\begin{array}{l}22 \text { of } 30, \\
73.33 \%\end{array}$ & $\begin{array}{l}\text { Both CD- } \\
\text { Infectious } \\
\text { (Influenza, } \\
\text { Pneumonia), } \\
\text { NCD-Chronic } \\
\text { (Asthma) }\end{array}$ \\
\hline $\begin{array}{l}\text { Various } \\
\text { communicable } \\
\text { diseases }\end{array}$ & {$[22]$} & $3.33 \%$ & NCD-Chronic \\
\hline $\begin{array}{l}\text { Various } \\
\text { infectious disease }\end{array}$ & [29] & $3.33 \%$ & CD-Infectious \\
\hline $\begin{array}{l}\text { Cardiovascular } \\
\text { disease, heart } \\
\text { disease }\end{array}$ & {$[24][35][40]$} & $10 \%$ & NCD-Chronic \\
\hline $\begin{array}{l}\text { Urinary tract } \\
\text { problems }\end{array}$ & [24] & $3.33 \%$ & CD-Infectious \\
\hline Skin problem & $\begin{array}{l}{[24][27][28]} \\
{[36]}\end{array}$ & $13.33 \%$ & CD-Infectious \\
\hline $\begin{array}{l}\text { Heat stroke/ } \\
\text { attack, Sunlight } \\
\text { effects }\end{array}$ & $\begin{array}{l}{[25][28][30]} \\
{[44][49]}\end{array}$ & $16.67 \%$ & NCD-Chronic \\
\hline $\begin{array}{l}\text { Diabetic/ diabetes } \\
\text { mellitus }\end{array}$ & $\begin{array}{l}{[24][25][26]} \\
{[48]}\end{array}$ & $13.32 \%$ & NCD-Chronic \\
\hline Hypertension & [25] [48] & $6.67 \%$ & NCD-Chronic \\
\hline Gastroenteritis & {$[25][27][36]$} & $10 \%$ & NCD-Chronic \\
\hline Dehydration & {$[25][30]$} & $6.67 \%$ & NCD-Chronic \\
\hline $\begin{array}{l}\text { Meningococcal } \\
\text { disease }\end{array}$ & {$[28]$} & $3.33 \%$ & CD-Infectious \\
\hline Musculoskeletal & [24] [36] & $6.67 \%$ & NCD-Chronic \\
\hline $\begin{array}{l}\text { Diarrhea and } \\
\text { jaundice }\end{array}$ & {$[42]$} & $3.33 \%$ & CD-Infectious \\
\hline $\begin{array}{l}\text { Traffic accidents } \\
\text { and Trauma }\end{array}$ & {$[35]$} & $3.33 \%$ & NCD-Chronic \\
\hline
\end{tabular}

Health problems or diseases are categorized into two main classes, namely communicable or infectious diseases and noncommunicable or chronic diseases. According to the studies, as shown earlier in Table I and Table III, $62.5 \%$ are noncommunicable diseases and $37.5 \%$ are communicable diseases, as shown in Fig. 5, encountered by the pilgrims during Hajj.

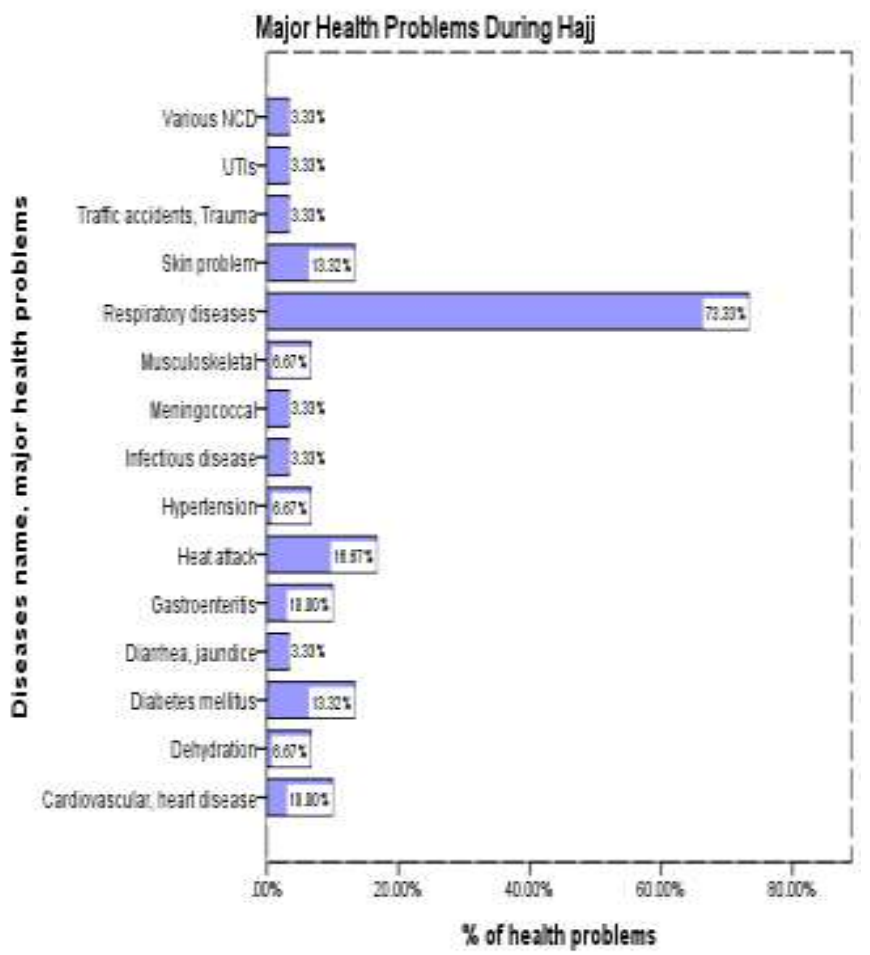

Fig. 4. Percentage of studies according to major health problems

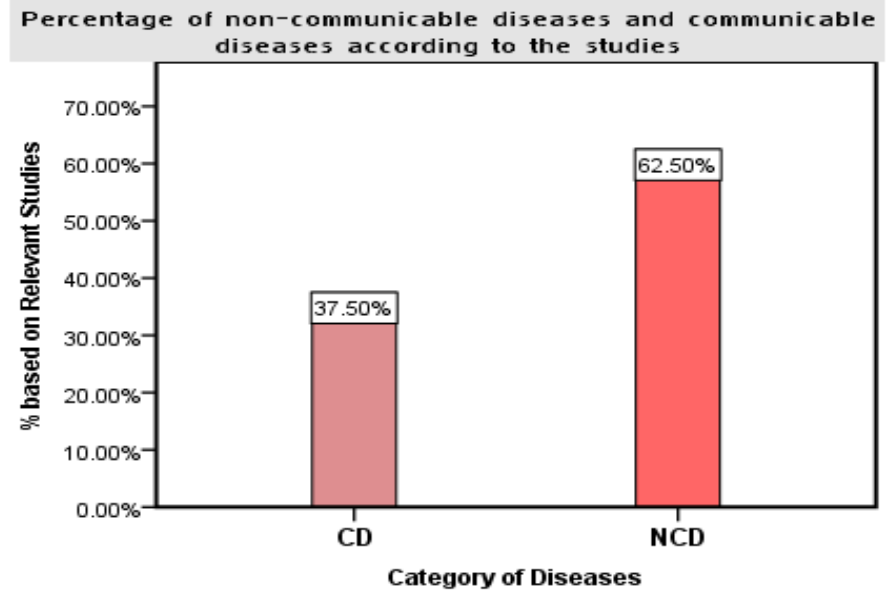

Fig. 5. Percentage of non-communicable diseases and communicable diseases according to the studies 


\section{DISCUSSION OF THE RESEARCH}

This concise review is intended to present a structured analysis of published articles of health problems and emergency situation over the past ten years with regards to Hajj pilgrim. Health problems and their challenges for pilgrims during the pilgrimage, related outcomes, and suggestions for future investigators are highlighted in this study.

Respiratory Tract Infections were considered as the predominant clinical health patterns which encountered by Hajj pilgrims. It continues to be the increasing burden of diseases among Hajj pilgrims, but there is still a lack of studies being conducted to overcome these problems. Researchers had identified respiratory diseases as the most common cause of hospital admission (52.5\%) during Hajj, with pneumonia being the leading reason for hospitalization. A prospective study was conducted in two different hospitals during Hajj 2011 [24]. The overall mortality rate in the ward among pilgrims with pneumonia was $2.4 \%$ and in the ICU was $21.45 \%$ during Hajj period 2004-2013 which is similar to the mortality rates in West [23]. Out of 30 selected studies, 22 of articles had concluded that respiratory diseases includes pneumonia, influenza and asthma $73.33 \%$ were the main health problems encountered by the pilgrims during Hajj [23] [24] [26] [27] [28] [31] [32] [33] [34] [35] [36] [37] [38] [39][41][42] [43][45] [46] [47][50] [51].

Meningococcal Disease: A crowded environment with high humidity and dense air pollution are the main reasons for a meningococcal disease which is defined as an infection as high as $3.33 \%$ among all the diseases encountered by pilgrims during Hajj [28].

Skin Infections: Bacterial skin infection is one of the pilgrims' health problems where Makkah is one of the hottest places in the world with the temperature range of 38 to $42^{\circ} \mathrm{C}$ during Hajj. The studies revealed that $23.6 \%$ dermatitis and $11.2 \%$ pyoderma patients were reported during Hajj. Among 80 pyoderma cases, $52.5 \%$ were primary pyoderma where impetigo was the leading causes for primary pyoderma. Whereas, $47.5 \%$ were secondary pyoderma led by Staphylococcus aureus responsible as main causative agents and followed by Streptococcus pyogenes [24] [27] [28] [36].

Environmental Heat Injury: The main factor of heat stress during Hajj as revealed from the literature are extreme summer temperature, direct and long time heat exposure from the sun, heat from vehicles and internal heat which lead to heat exhaustion or heat stroke among pilgrims [25] [28] [30] [44] [49].

Cardiovascular Diseases: Over the past few years, the study revealed cardiovascular disease with hypertension is one of the important causes of pilgrims' intensive care unit-ICU admission with high mortality rate [24] [40]. During 2002 Hajj, the percentage of cardiovascular diseases was 45.8 [35].

Gastrointestinal infections: Major food-borne outbreaks of gastroenteritis with high mortality rates are common at all religious festivals, including the Hajj [25] [27] [36].
Blood-borne diseases: To shave head during Hajj is compulsory which leads to transmission of blood-borne diseases including hepatitis B, C, and HIV. Illegal unlicensed barbers continue to operate the act whether the Saudi Ministry of health-MoH promotes and encourages all pilgrims to receive hepatitis $B$ vaccination before travel to Hajj [22, 29].

Malaria: Although WHO classifies Saudi Arabia as a low, geographically restricted malaria transmission area and since 2008 has been listed as being in the elimination stage of the program, but the risk during Hajj is still exist. In 2011 Hajj season 19 cases of $\mathrm{P}$ vivax malaria was reported where $75 \%$ such cases found among the Indian and Ethiopian pilgrims. In 2012, 48 cases of malaria were recorded in Makkah and 78 cases were recorded in Madinah among Pakistan, Nigeria, Guinea, India, Mouritania, Chad, Mali, Afghanistan, Somalia, Ethiopia, Yemen and Ivory Coast pilgrims.

Trauma risks: During Hajj trauma is one of the major causes of morbidity and mortality. In a prospective study of 713 trauma patients, who were injured while performing Hajj, presenting to the emergency room, 248 (35\%) were admitted to surgical departments and intensive care. The most common surgical presentations were orthopedic and neurosurgical [35]. For a large part of the Hajj, pilgrims travel either by foot, walking through or near dense traffic, or in vehicles themselves.

Fire-related injury: In 1997, fire devastated the Mina area when makeshift tents were set ablaze by open stoves since banned at the Hajj. There were 343 deaths and more than 1500 estimated casualties. Since then all makeshift tents have been replaced by permanent fiberglass installations. At Hajj time, Teflon-coated awnings are added, and the aluminum frames remain in place the rest of the year. No pilgrim is permitted to set up his own tent. Additionally, pilgrims are not allowed to cook food at Mina. Smoking is forbidden during the Hajj by Islamic teaching, thus reducing the risk of a naked flame. Continuous public education is being undertaken to further reduce fire risk.

Environmental heat injury: Heat exhaustion and heatstroke are a leading cause of morbidity and mortality during the Hajj, particularly in summer. Temperatures in Mecca can rise higher than $45^{\circ} \mathrm{C}$. Lack of acclimatization, arduous physical rituals, and exposed spaces with limited or no shade, produces heatstroke in many pilgrims. Adequate fluid intake and seeking shade is essential. Supplicating pilgrims might not notice the dangers of extreme heat exposure until their symptoms are pronounced. Water mist sprayers operate regularly in the desert at Arafat, a time of high risk for heatstroke, when many stand for long hours during the day. Performing rituals at night, using umbrellas, seeking shade, and wearing high-SPF sunblock creams are all advisable and permissible during the Hajj. Children accompanying their parents must be specially protected. The timings of rites are flexible and acceptable at the pilgrim's convenience - it is key that pilgrims are aware of this since, through fear of committing errors, they might not make sensible choices in completing their rituals [25] [28] [30] [44] [49]. Although the Hajj is not due to fall in the summer for 
several years, Saudi winters are warmer $\left(35-50^{\circ} \mathrm{C}\right)$ than most pilgrims will be used to, and they must seek shade and drink plenty of fluid during their rites.

Occupational hazards of abattoir workers: Abattoir workers at the Hajj are exposed to unique traumatic risks. Over a million cattle are slaughtered each Hajj, up to half a million before noon on the $10^{\text {th }}$ day of the Hajj. In one study, 298 emergency visits for hand injury were treated in Mecca over four Hajj seasons. More than $80 \%$ were injuries from animal slaughter; many avoidable injuries were sustained by lay people and not trained abattoir workers. Pilgrims need to be assured that professional slaughtering arrangements are easily available at the Hajj, and far safer.

\section{CONCLUSION}

Both communicable and non-communicable health issues are the most common health problems encountered by pilgrims during Hajj. But, due to lack of existing studies associated with this research area, a definite conclusion could not be made. However, our findings demonstrated the necessity of new research to find solutions to pilgrims' health problems during Hajj.

\section{ACKNOWLEDGEMENT}

This study obtained no funding. Authors do not have any conflict of interest.

\section{REFERENCES}

[1] Gatrad AR, Sheikh A. Hajj: journey of a lifetime. BMJ 2005; 330: 13337.

[2] Kraemer JL. The Hajj: the Muslim pilgrimage to Mecca and the holy places. Hist Religions 2000; 40: 198-203.

[3] WHO report on Hajj: http://www.who.int/csr/ITH_final.pdf

[4] Ministry of Health, KSA guideline on Hajj: https://www.moh.gov.sa/en/Hajj1436/Awareness/Documents/DlelHajE.pdf

[5] "1,384,941 foreign pilgrims participated in Hajj". Royal Embassy of Saudi Arabia. 22 September 2015. Retrieved 26 Sep 2015.

[6] Ministry of Hajj, Saudi Arabia. Supreme Hajj Committee. http://www.hajinformation.com/main/u1.htm (accessed May-June, 2016).

[7] Central Department of Statistics and Information. http://www.cdsi.gov.sa/english/ (accessed May-June, 2016).

[8] M Khogali, Epidemiology of Heat Illnesses During the Makkah Pilgrimages in Saudi Arabia, International Journal of Epidemiology, Vol. 12, No. 3, 1983,12: 267-273

[9] Ghaznawi HI, Ibrahim MA. Heat stroke and heat exhaustion in pilgrims performing the Haj (annual pilgrimage) in Saudi Arabia. Ann Saudi Med 1987;7:323-6.

[10] Z A Memish, S J N McNabb, F Mahoney, F Alrabiah, N Marano, Q A Ahmed, J Mahjour, R A Hajjeh, P Formenty, F H Harmanci, H El Bushra T M Uyeki, M Nunn, N Isla, M Barbeschi, and the Jeddah Hajj Consultancy Group, Establishment of public health security in Saudi Arabia for the 2009 Hajj in response to pandemic influenza A H1N1, Lancet 2009; 374: 1786-91

[11] Memish ZA, Ahmed QA. Mecca-bound: the challenges ahead. J Travel Med 2002; 9: 202-10.

[12] Rashid H, Shafi S, Haworth E, et al. Viral respiratory infections at the Hajj: comparison between UK and Saudi pilgrims

[13] Madani TA, Ghabrah TM, Albarrak AM, et al. Causes of admission to intensive care units in the Hajj season of the Islamic year 1424 (2004). Ann Saudi Med. 27; 2007: 101-105. Available from: URL: http://www.researchgate.net/publication
/6451514_Causes_of_admission_to_intensive_care_units_in_the_Hajj_p eriod_of_the_Islamic_year_1424_(2004)

[14] Shuja Shafi, Ziad A Memish , Abdul Rashid Gatrad, Aziz Sheikh, Hajj 2006: communicable disease and other health risks and current official guidance for pilgrims, Eurosurveillance, 15 December 2005

[15] Ahmed Q, Arabi Y, Memish ZA. Health risks at the Hajj. Lancet 2006;367(9515):1008-15

[16] Mandourah Y, Al-Radi A, Ocheltree AH, Ocheltree SR, Fowler RA. Clinical and temporal patterns of severe pneumonia causing illness during Hajj. BMC Infect Dis. 12; 2012: 117. Available from: URL: http://www.biomedcentral.com/1471-2334/12/117

[17] Gatrad AR, Shafi S, Memish ZA, Sheikh A. Hajj and the risk of influenza. Br Med J. 33; 2006: 1182-1183. Available from: URL: http://www.bmj.com/content/333/7580/1182.full.pdf + html

[18] El-Sheikh SM, El-Assouli SM, Mohammed KA, Albar M. Bacteria and viruses that causes respiratory tract infections during the pilgrimage (Haj) season in Makkah, Saudi Arabia. Trop MedInt Health. 3(3); 1998: 20509. Available from: URL: http://www.researchgate.net/publication/13691513_Bacteria_and_viruse s_that_cause_respiratory_tract_infections_during_the_pilgrimage_(Haj) _season_in_Makkah_Saudi_Arabia

[19] Abdulrahman R. Bakhsh, MBBS, EMDM, Abdulfattah I. Sindy, MBBS, Mostafa J. Baljoon, PhD, Khalid O. Dhafar, FRCS, FACS, Zohair J. Gazzaz, MBChB, PhD, Mukhtiar Baig, MBBS, $\mathrm{PhD}$, Basma A. Deiab, PhD, and Fauzea T. Al Hothali, DN, BSN, Diseases pattern among patients attending Holy Mosque (Haram) Medical Centers during Hajj 1434 (2013), Saudi Med J. 2015 Aug; 36(8): 962-966.

[20] Meysamie A, Ardakani HZ, Razavi SM, Doroodi T. (2006). Comparison of mortality and morbidity rates among Iranian pilgrims in Hajj 2004 and 2005. Saudi Med J. 27 (7): 1049- 1053. Available from: URL: smj.psmmc.med.sa/index.php/smj/article/viewFile/3733/1507

[21] British Broadcasting Corporation (BBC) News. 2006. Available from: URL: http://news.bbc.co.uk/2/hi/middle_east/6164337.stm

[22] Ziad A Memish, Habida Elachola, Mujeeb Rahman, Samba Sow, Nawfal Aljerian, Abdullah Assir, Objection to chronic disease based restrictions during the Hajj, www.thelancet.com Vol 387 April 23, 2016

[23] Bader Hamza Shiraha, Syed Husham Zafar b, Olayan Ali Alferaidi b, Abdul Momin Muhammad Sabir b, Mass-gathering medicine (Hajj Pilgrimage in Saudi Arabia): The clinical pattern of pneumonia among pilgrims during Hajj, Journal of Infection and Public Health (2016) April

[24] Murtaza Ali Gowa, Syed Ali Ammar, Ahmed Ali, Syed Muhammad Kashif Kazmi, Rana Farrukh, Ali Abbas Mohsin Ali \& Syed Muhammad Mustahsan, Health Issues confronting Muslims on Hajj, International Journal of Endorsing Health Science Research www.aeircedu.com Volume 3 Issue 2, July 2015

[25] Sindy AI, Baljoon MJ, Zubairi NA, Dhafar KO, Gazzaz ZJ, Deiab BA, et al. Pattern of patients and diseases during mass transit: The day of Arafat experience. Pak J Med Sci 2015;31(5):1099-1103. doi: http://dx.doi.org/10.12669/pjms.315.8017

[26] Habsah Hasan, Zakuan Zainy Deris, Siti Amrah Sulaiman, Mohd Suhaimi Abdul Wahab, Nyi Nyi Naing, Zulkefle Ab Rahman, Nor Hayati Othman, Effect of Influenza Vaccination on Acute Respiratory Symptoms in Malaysian Hajj Pilgrims, Journal of Immigrant and Minority Health, August 2015, Volume 17, Issue 4, pp 1114-1119

[27] Abdulrahman R. Bakhsh, MBBS, EMDM, Abdulfattah I. Sindy, MBBS, Mostafa J. Baljoon, PhD, Khalid O. Dhafar, FRCS, FACS, Zohair J. Gazzaz, MBChB, PhD, Mukhtiar Baig, MBBS, $\mathrm{PhD}$, Basma A. Deiab, PhD, and Fauzea T. Al Hothali, DN, BSN, Diseases pattern among patients attending Holy Mosque (Haram) Medical Centers during Hajj 1434 (2013), Saudi Med J. 2015 Aug; 36(8): 962-966.

[28] Nurul Diana Dzaraly, Nor Iza A. Rahman, Nordin Bin Simbak, Suhaimi Ab. Wahab, Omar Osman, Salwani Ismail, Mainul Haque, Patterns of Communicable and Non-Communicable Diseases in Pilgrims during Hajj, Research J. Pharm. and Tech. 7(9): Sept. 2014 Page 1052-1059 
[29] Ziad A Memish, Alimuddin Zumla, Rafat F Alhakeem, Abdullah Assiri, Abdulhafeez Turkestani, Khalid D Al Harby, Mohamed Alyemni, Khalid Dhafar, Philippe Gautret, Maurizio Barbeschi, Brian McCloskey, David Heymann, Abdullah A Al Rabeeah, Jaffar A Al-Tawfi q, Hajj: infectious disease surveillance and control, Lancet mass gatherings medicine 1, www.thelancet.com Vol. 383 June 14, 2014

[30] Habida Elachola, Abdullah M. Assiri, Ziad A. Memish, Sun protection during the Hajj mass-gathering - 2013, Travel medicine and infectious disease, Elsevier, November December, 2014Volume 12, Issue 6, Part B, Pages 783-784 DOI: http://dx.doi.org/10.1016/j.tmaid.2014.09.005

[31] Osamah Barasheed, Harunor Rashid, Mohammad Alfelali, Viral respiratory infections among Hajj pilgrims in 2013, VIROLOGICA SINICA 2014, 29 (6): 364-371, DOI 10.1007/s12250-014-3507-x

[32] Samir Benkouiten, Rémi Charrel, Khadidja Belhouchat, Tassadit Drali, Antoine Nougairede, Nicolas Salez, Ziad A. Memish, Malak Al Masri, Pierre-Edouard Fournier, Didier Raoult, Philippe Brouqui, Philippe Parola, and Philippe Gautret, Respiratory Viruses and Bacteria among Pilgrims during the 2013 Haji, Emerging infectious diseases, Volume 20, Number 11-November 2014, DOI: 10.3201/eid2011.140600

[33] Samir Benkouiten, Rémi Charrel, Khadidja Belhouchat, Philippe Gautret et. al. Circulation of Respiratory Viruses Among Pilgrims During the 2012 Haji Pilgrimage, Clinical Infectious Diseases (2013) 57 (7):992-1000.doi: 10.1093/cid/cit446First published online: July 9, 2013

[34] Al-Tawfiq JA, Zumla A, Memish ZA. Respiratory tract infections during the annual Haij: potential risks and mitigation strategies. Current Opinion in Pulmonary Medicine, Infectious diseases, 2013; 19:192-7.

[35] Abdullah Al Shimemeri, Cardiovascular disease in Hajj pilgrims, Journal of the Saudi Heart Association, Volume 24, Issue 2, April 2012, Pages 123-127

[36] Alzahrani AG, Choudhry AJ, Al Mazroa MA, Turkistani AHM, Nouman GS, Memish ZA. Pattern of diseases among visitors to Mina health centers during the Hajj season, $1429 \mathrm{H}$. Journal of Infection and Public Health. 5; 2012: 22-34. Available on http: //Elsevier.com/locate/jiph

[37] Mandourah Y, Al-Radi A, Ocheltree AH, Ocheltree SR, Fowler RA. Clinical and temporal patterns of severe pneumonia causing illness during Hajj. BMC Infect Dis. 12; 2012: 117. Available from: URL: http://www.biomedcentral.com/1471-2334/12/117

[38] Memish Z.A, Assiri A.M, Hussain R, Alomar I, Stephens G. Detection of respiratory viruses among pilgrims in Saudi Arabia during the time of a declared Influenza A(H1N1) Pandemic. J Travel Med. 19(1); 2012: 15-2. Available from: URL: http://www.researchgate.net/publication/51922829_The_prevalance_of_ respiratory_viruses_among_healthcare_workers_serving_pilgrims_in_M akkah_during_the_2009_influenza_A_(H1N1)_pandemic/file/32bfe50e 495caab336.pdf

[39] Afagh Moattari, Amir Emami, Mohsen Moghadami, Behnam Honarvar, Influenza viral infections among the Iranian Hajj pilgrims returning to Shiraz, Fars province, Iran. Influenza and Other Respiratory Viruses 6(601), 2012, e77-e79

[40] WH Almalki, The_prevalence_of_cardiovascular diseases_and_role_of protective measures among hajj pilgrims 1432)_2011,
Pakistan Journal of Pharmacology Vol.29, No.2, July 2012, pp.29-34

[41] Alborzi A, Aelami MH, Ziyaeyan M, Jamalidoust M, Moeini M, Pourabbas B, Abbasian A. Viral etiology of acute respiratory among Iranian Hajj pilgrims. J. Travel Med. 16(4); 2009: 239-242. Available on http://onlinelibrary.wiley.com/doi/10.1111/j.1708-305.2009.00301.x/pdf

[42] Islam Saeed, Jawad Mofleh, Hafiz, Iqbal, Occurrence of acute respiratory infection, diarrhea and jaundice among Afghan pilgrims, 2010, Source: PubMed, December 2012 DOI: 10.1016/j.jegh.2012.11.003

[43] Asghar AH, Ashshi AM, Azhar EI, Bukhari SZ, Zafar TA, Momenah AM. Profile of bacterial pneumonia during Hajj. Indian J Med Res. 133; 2011: 510-513. Available from: URL: http://www.researchgate.net/publication/51175189_Profile_of_ba cterial_pneumonia_during_Hajj

[44] Mimish, L. Electrocardiograph findings in heat stroke and exhaustion: A study on Makkah pilgrims. J Saudi Heart Assoc. 24; 2012: 35-39. Available

from: URL:http://www.sciencedirect.com/science/article/pii/S1016731511002 119

[45] Mirza TA, Fillimban A, Maimini O, Khiyat EY, Dhafar KO, Farooq MU, Gazzaz ZJ. Predictors of asthma severity during the pilgrimage to Mecca (Hajj). Pol Arch Med Wewn. 121 (10); 2011: 327-332. Available from: URL:http://allegro.cartel color.com/sites/default/files/pamw_201110_57-Farooq_0.pdf

[46] Zakuan Zainy Deris, Habsah Hasan, Mohd Suhaimi Ab. Wahab, Siti Amrah Sulaiman, Nyi Nyi Naing and Nor Hayati Othman, The association between pre-morbid conditions and respiratory tract manifestations amongst Malaysian Hajj pilgrims, Tropical Biomedicine 27(2): 294-300 (2010)

[47] Alborzi A, Aelami MH, Ziyaeyan M, Jamalidoust M, Moeini M, Pourabbas B, Abbasian A. Viral etiology of acute respiratory among Iranian Hajj pilgrims. J. Travel Med. 16(4); 2009: 239-242. Available on http://onlinelibrary.wiley.com/doi/10.1111/j.17088305.2009.00301.x/pdf

[48] P. Gautret1, W. Yong1,2, G. Soula1,3, J. Gaudart4, J. Delmont1,3, A. Dia1, P. Parola1 and P. Brouqui1, Incidence of Hajj-associated febrile cough episodes among French pilgrims: a prospective cohort study on the influence of statin use and risk factors, Journal of Clinical Microbiology and Infect 2009; 15: 335-340

[49] Gautret P, Soula G, Delmont J, Parola P, Brouqui P. Common health hazards in French pilgrims during the Hajj of 2007. A prospective cohort study. J Travel Med. 16(6): 2009: 377-81. Available from: URL: www.ncbi.nlm.nih.gov/pubmed/19930376

[50] Rashid H, Shafi, Booy, RS, El Bashir H, Ali K, Zambon, MC, et al. Influenza and respiratory syntical virus infection in British pilgrims. Emerg Health Threats J. 1(2); 2008: 2-5. Available from: URL www.eht-journal .org

[51] Rashid H, Shafi S, Haworth, E, El Bashir H, Memish ZA, Sudhanva M, et al.. Viral respiratory infections at the Hajj: Comparison between UK and Saudi pilgrims. Clin Microbiol Infect. 14; 2008: 569-74. Available from: URL: http://onlinelibrary.wiley.com/doi/10.1111/j.14690691.2008.01987.x/pdf on 12/8/14 